\title{
Estudo do uso e cobertura da terra no Parque Estadual Mãe Bonifácia na Cidade de
}

\section{Cuiabá-MT}

\author{
Study of land use and land cover in the Mãe Bonifácia State Park in the City of Cuiabá-MT \\ Estudio de uso y cobertura del suelo en el Parque Estatal Mãe Bonifácia en la Ciudad de Cuiabá-
}

Luciene Toledo e Almeida

ORCID: https://orcid.org/0000-0002-6676-2177 Instituto Federal de Educação, Ciência e Tecnologia de Mato Grosso, Brasil E-mail: luhhtalmeida@gmail.com

Karoline Assuero Cintra Ferreira ORCID: https://orcid.org/0000-0003-3870-3126 Instituto Federal de Educação, Ciência e Tecnologia de Mato Grosso, Brasil E-mail:assuero.engflorestal@gmail.com

Noelto da Cruz Teixeira

ORCID:https://orcid.org/0000-0002-4728-9556 Instituto Federal de Educação, Ciência e Tecnologia de Mato Grosso, Brasil E-mail: noelto.teixeira@cba.ifmt.edu.br

Norka da Silva Albernaz Marcilio

ORCID: https://orcid.org/0000-0002-9942-7551 Instituto Federal de Educação, Ciência e Tecnologia de Mato Grosso, Brasil E-mail: norka.albernaz@gmail.com

Geraldo Antônio Gomes de Almeida ORCID: https://orcid.org/0000-0002-4868-4224 Instituto Federal de Educação, Ciência e Tecnologia de Mato Grosso, Brasil E-mail: geraldo.almeida@cba.ifmt.edu.br

Patrícia Mota Rausch

ORCID: https://orcid.org/0000-0003-2959-2081 Instituto Federal de Educação, Ciência e Tecnologia de Mato Grosso, Brasil E-mail: patricia.rausch@cba.ifmt.edu.br

Vanderley Severino

ORCID: https://orcid.org/0000-0002-0623-2246 Instituto Federal de Educação, Ciência e Tecnologia de Mato Grosso, Brasil

E-mail: Vanderley.santos@cba.ifmt.edu.br

Victor Hugo de Morais Danelichen ORCID: https://orcid.org/0000-0003-4791-3301 Universidade de Cuiabá, Brasil

E-mail: danelichen@ @isica.ufmt.br

Guilherme Barros Seixas

ORCID: https://orcid.org/0000-0002-1667-6086

Instituto Federal de Educação, Ciência e Tecnologia de Mato Grosso, Brasil

E-mail:guilherme.seixas@cba.ifmt.edu.br

\begin{abstract}
Resumo
O uso e cobertura da terra podem ser analisados usando o Índice de Vegetação por Diferença Normalizada (NDVI) e a Temperatura da Superfície da Terra (TST). Em áreas densamente povoadas, o aumento da temperatura do ar devido ao efeito da ilha de calor urbana pode prejudicar o conforto térmico e a saúde dos cidadãos. O parque urbano é um critério importante para avaliar a responsabilidade do meio ambiente nas cidades, especialmente aquelas de clima tropical. Nesse sentido, o presente trabalho teve como objetivo estudar a relação entre uso e ocupação do solo, NDVI e TST no Parque Estadual Mãe Bonifácia (PEMB) e seu entorno na cidade de Cuiabá, Mato Grosso, nos anos de 2013 e 2020 com o uso de técnicas de sensoriamento remoto e Sistema de Informação Geográfica (SIG). Os resultados mostraram relações inversas entre os valores de NDVI e TST no período considerado, evidenciando os benefícios microclimáticos da área de estudo. Conclui-se, assim, que existe uma relação entre os padrões de ocupação do solo e o clima local. Dessa forma, o planejamento da gestão administrativa de áreas verdes nas cidades pode otimizar o conforto térmico da população, garantindo um ambiente saudável.
\end{abstract}

Palavras-chave: Sensoriamento remoto; Landsat 8; Conforto ambiental. 


\begin{abstract}
Land use and land cover can be analyzed using the Normalized Difference Vegetation Index (NDVI) and the Earth Surface Temperature (TST). In densely populated areas, the increase in air temperature due to the urban heat island effect can harm the thermal comfort and health of citizens. Urban park is an important criterion to assess the responsibility of the environment in cities, especially those with a tropical climate. In this sense, the present work aimed to study the relationship between land use and land cover, NDVI and TST in the Mãe Bonifácia State Park (PEMB) and its surroundings in the city of Cuiabá, Mato Grosso, in the years 2013 and 2020 with the use of remote sensing techniques and Geographic Information System (GIS). The results showed inverse relationships between the values of NDVI and TST in the period considered, highlighting the microclimatic benefits of the study area. It is thus concluded that there is a relationship between land occupation patterns and the local climate. In this way, planning the administrative management of green areas in cities can optimize the thermal comfort of the population, ensuring a healthy environment.
\end{abstract}

Keywords: Remote sensing; Landsat 8; Environmental comfort.

\title{
Resumen
}

El uso de la tierra y la cobertura de la tierra se pueden analizar usando el Índice de Vegetación de Diferencia Normalizada (NDVI) y la Temperatura de la Superficie de la Tierra (TST). En áreas densamente pobladas, el aumento de la temperatura del aire debido al efecto isla de calor urbano puede perjudicar el confort térmico y la salud de los ciudadanos. El parque urbano es un criterio importante para evaluar la responsabilidad del medio ambiente en las ciudades, especialmente aquellas con clima tropical. En este sentido, el presente trabajo tuvo como objetivo estudiar la relación entre uso y cobertura del suelo, NDVI y TST en el Parque Estatal Mãe Bonifácia (PEMB) y sus alrededores en la ciudad de Cuiabá, Mato Grosso, en los años 2013 y 2020 con el uso de técnicas de teledetección y Sistema de Información Geográfica (SIG). Los resultados mostraron relaciones inversas entre los valores de NDVI y TST en el período considerado, destacando los beneficios microclimáticos del área de estudio. Por tanto, se concluye que existe una relación entre los patrones de ocupación de la tierra y el clima local. De esta forma, planificar la gestión administrativa de las áreas verdes en las ciudades puede optimizar el confort térmico de la población, asegurando un medio ambiente saludable.

Palabras clave: Teledetección; Landsat 8; Confort ambiental.

\section{Introdução}

Atualmente, a questão ambiental, embora, muito discutida e de importância e relevância global, às eventuais consequências dos desequilíbrios, ainda, é emergente quanto à sua aplicabilidade de forma quali-quantitativa, o que se verifica um caminho de gestão administrativa e ambiental ainda inacabado.

Diante disso, antecipa-se o valor potencial das informações homem-natureza onde uma nova ordem socializada entra em vigor, tornando um desafio de gestão que precisa ser enfrentado para um melhor conforto da população para os próximos séculos.

$\mathrm{Na}$ evolução das atividades antrópicas, observa-se que as cidades cresceram junto às necessidades das organizações sociais. Segundo relatório da ONU (2014), 54\% da população mundial vive em áreas urbanas e com projeções que podem chegar a 66\% até 2050 (Martins, 2018). Assim, ações do homem sobre a natureza altera o clima local e, seus efeitos são percebidos pela população, em geral, pelas questões relativas ao conforto térmico, umidade relativa do ar, impactos pluviais e outros efeitos que desequilibram a vida nas cidades.

Sabe-se, portanto, que a urbanização altera significativamente o clima, e que essas mudanças variam de acordo com a intensidade do uso e da ocupação da terra, além, das características geológicas do lugar. Oliveira e Bitar (2009) citam que as cidades brasileiras estão passando por um período de acentuada urbanização, o que reflete negativamente na qualidade de vida de seus habitantes. Diferentes densidades de construção, possuem uma grande capacidade de armazenar calor, afetando o clima urbano (Wall \& Waterman, 2012).

Em centros urbanos densamente povoados, o aumento da temperatura do ar devido ao efeito da ilha de calor urbano pode prejudicar o conforto térmico e a saúde dos cidadãos. Grandes parques urbanos podem mitigar o efeito de ilhas de calor e melhorar o conforto térmico, especialmente, nos meses mais quentes do ano, quando as mudanças de temperatura são mais 
perceptíveis. Aram et al., (2020), ainda, ressaltam que a intensidade do efeito de resfriamento varia conforme a distância da borda dos parques.

Segundo Duarte (2010), a cobertura vegetal e os cursos d'água espalhados pela cidade caracterizam o balanço de energia em áreas urbanizadas, atuando como amenizadores das condições microclimáticas locais. Observando a relação entre os padrões de ocupação da terra e os microclimas urbanos, pode-se traçar melhorias nas condições de conforto ambiental (Martins, 2018). Assim, também, o papel importantíssimo que a flora desempenha no balanço de energia, particularmente no caso de cidades tropicais com expressiva continentalidade (Monteiro, 2015).

Neste sentido, os parques urbanos apresentam um papel fundamental no melhoramento do clima, possibilitando a recreação e lazer, além de proteger a mata nativa e os maciços vegetais existentes. A temperatura é a principal variável para analisar a qualidade dos parques, pois, a redução da amplitude térmica contribui para o bem-estar da população (Cunha et al., 2017).

Diversos trabalhos foram desenvolvidos com esta temática com o intuito de compreender a atuação do clima local e a valorização do papel das florestas urbanas (Silva \& Silva, 2016; Rodrigues, Pasqualetto \& Garção, 2017; Melo \& Dias, 2019).

Considerando o exposto, o Índice da Vegetação por Diferença Normalizada (NDVI), proposto por Rouse et al., (1973), tem sido bastante utilizado para realçar o vigor da vegetação e sua evolução cronológica, a partir da reflectância das bandas do visível e do infravermelho próximo, extraída das imagens satelitais. O NDVI tornou-se um índice espectral padrão para estudar o uso e cobertura da terra e, comporta-se de maneira inversa em relação a TST, em que, as temperaturas menores, geralmente ocorrem em áreas de vegetação mais densa e vigorosas e valores altos em vegetação menos densa e solos expostos. Por outro lado, para faixas de temperaturas mais elevadas em áreas com vegetação menos densa, torna-se passível a ocorrência de valores reduzido para o NDVI (Mwangi; Karanja; Kamau, 2018).

No estudo de microclimas, envolvendo as informações do uso e cobertura da terra, NDVI e TST extraídas das imagens de sensoriamento remoto em ambientes de SIG tem relevante destaque, na medida, em que é possível estabelecer relações entre as variáveis biofísicas e antrópicas (Lopes et al., 2018; Azambuja, et al., 2018; Pinheiro \& Silva, 2019). Barros, Farias e Marinho (2020) apontam que o sensoriamento remoto orbital, tornou-se uma ciência atrativa na medida em que as informações dos objetos da superfície terrestre são obtidas por aparelhos distantes dos alvos e permite a redução dos custos e apresentam maior rapidez na obtenção da informação.

Desta maneira, a tecnologia de sensoriamento remoto oferece oportunidades importantes para detectar e monitorar mudanças ambientais em escala local, regional ou global (Ochtyra et al., 2020), assim também, importante ferramenta para a geração e produção de conhecimentos e, igualmente quanto a integração dos mesmos como subsídio no planejamento urbano (Eduvirgem et al., 2019).

Diante do exposto, este trabalho objetiva por meio de técnicas de sensoriamento remoto e SIG estimar e avaliar a relação entre a TST, NDVI e o uso e ocupação da terra no PEMB e em seu entorno dos anos de 2013 e 2020 na cidade de Cuiabá - MT, a fim de condicionar melhor a interpretação da temperatura no clima urbano na área de estudo.

\section{Materiais e Métodos}

\section{Área de Estudo}

A área de estudo está localizada no município de Cuiabá, região Centro Oeste do Brasil e na porção Sul do Estado de Mato Grosso, entre as coordenadas planas UTM, 594875 m e 597066 m Este e 8278379 m e 8276236 m Norte, referenciadas ao Datum global WGS 84, fuso 21 do hemisfério sul, conforme a Figura 1. Possui uma área de $0,775 \mathrm{Km}^{2}$, tendo como limites as avenidas Miguel Sutil, Senador Filinto Muller e a rua Corsino do Amarante, (Cuiabá 2004; Ipdu, 2012). 

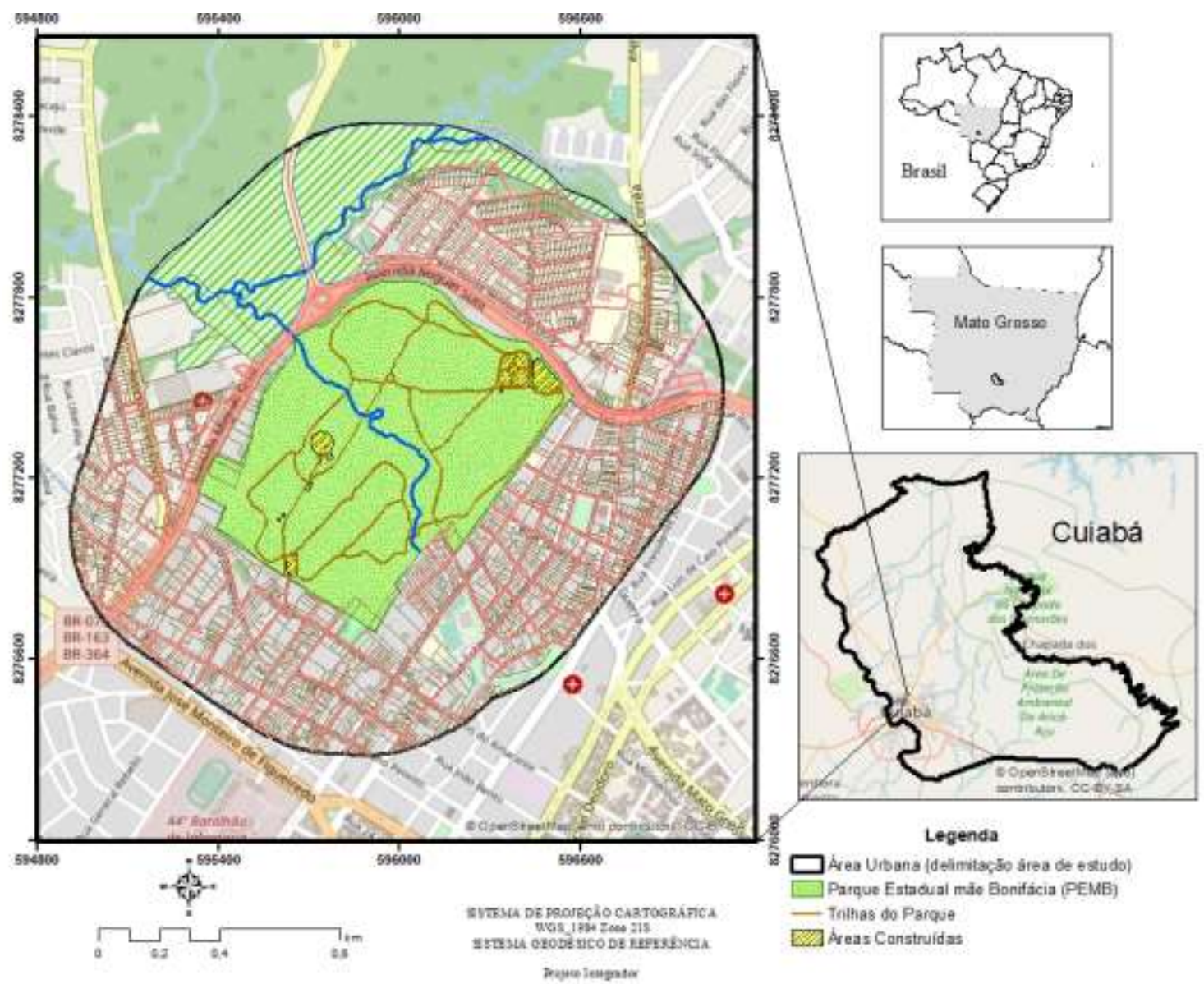

Fonte: Autores (2021).

O PEMB está inserido no Bioma Cerrado e na Depressão Cuiabana, onde predominam relevos de baixas amplitudes, com altitudes variando de 146 a 250 metros constituído por vegetação arbustiva e herbácea, apresentando resquícios da mata de galeria ao longo dos cursos dos rios e, designado como berçário das principais bacias hidrográficas brasileiras, permitindo o endemismo de vários espécimes (SEMA, 2013).

A significativa extensão da área e preservação florística do PEMB, possibilita a existência de uma fauna variada, como por exemplo, gambá (Didelphidae), sagüi (callithrichidae), sabiá (Mimidae), bem-te-vi (Turdidae), periquito (psittacidae) dentre outros (Cuiabá, 2021).

O crescimento acelerado na malha urbana da cidade de Cuiabá nos últimos anos associado com seu clima Tropical Continental Semiúmido, bem como, a redução da cobertura vegetal nativa em seu entorno pode-se considerar, atualmente, uma das regiões mais quente do Brasil. Segundo a classificação de Köppen, a região de Cuiabá apresenta um clima do tipo Aw com temperatura e precipitação média anual de $26^{\circ} \mathrm{C}$ e $1.360 \mathrm{~mm}$, respectivamente (Martins \& Romancini, 2005).

\section{Base de Dados e Processamento}

Neste trabalho foram utilizados os dados de reflectâncias extraídas pelos sensores Operational Land Imager (OLI) e Thermal Infrared Sensor (TIRS) a bordo do satélite Landsat 8 e, adquirida gratuitamente, no site da United States Geological Survey (USGS) no formato GeoTIFF, como mostra a Tabela 1. 
Tabela 1. Bandas espectrais utilizadas dos sensores OLI/TIRS.

\begin{tabular}{c|lcc}
\hline \multirow{2}{*}{ Sensores } & \multicolumn{1}{|c}{ Bandas } & $\begin{array}{c}\text { Intervalo } \\
\text { Espectral }\end{array}$ & $\begin{array}{c}\text { Resolução } \\
\text { Espacial (m) }\end{array}$ \\
\cline { 2 - 4 } & \multicolumn{1}{c}{ Banda 4 - Visível Vermelho } & $0.64-0.67$ & 30 \\
\multirow{3}{*}{ OLI } & Banda 5 - Infravermelho Próximo & $0.85-0.88$ & 30 \\
& Banda 6 - Infravermelho & $1.57-1.65$ & 30 \\
& Banda 8 - Pancromática & $0.50-0.68$ & 15 \\
\hline \multirow{2}{*}{ TIRS } & Banda 10- Infravermelho Termal & $10.60-11.19$ & 100 \\
\hline
\end{tabular}

Fonte: Autores (2021).

As imagens referem-se a órbita-ponto 226 -71, correspondentes as datas 16/07/2013 às 13:47 horas e 19/07/2020 às 13:45 horas e, tem uma resolução espacial de $30 \mathrm{~m}$ para o sensor OLI, exceto para a banda pancromática (Banda 8) de 15 m e 100m para a Banda 10 do sensor TIRS. Foi utilizado imagens do período seco de cada ano, devido à baixa cobertura de nuvens que possibilitou melhor visualização das cenas. As imagens adquiridas apresentaram correção das inconsistências atmosféricas, pois, a USGS, nos últimos anos, tem disponibilizado imagens com correção atmosférica de maior nível de qualidade, oferecendo uma melhoria do produto de refletância de superfície (Vermote et al., 2016).

O processamento foi realizado no ArcGis 10.5. Inicialmente, fez-se a reprojeção das imagens para a projeção Universal Transversa de Mercator (UTM) referenciada no Datum global WGS 1984, zona 21 no hemisfério sul. Os dados da distribuição da precipitação pluviométrica acumulada foram extraídos do site do Instituto Nacional de Meteorologia (INMET), Estação Cuiabá, código (WMO) A901, para os anos 2013 e 2020 e, organizados na planilha Excel.

\section{Uso e ocupação da terra, TST e NDVI}

O processamento das imagens e a obtenção da TST e NDVI foram desenvolvidos segundo o fluxograma da Figura 2.

Com a finalidade de melhorar a clareza visual das imagens para interpretação, criou-se as composições coloridas R6G5B4 com o uso das bandas 6, 5 e 4 do sensor multiespectral OLI, além de aumentar a resolução espacial com o uso da ferramenta espacial Pan-sharpened, que mescla a imagem pancromática da banda 8 de resolução 15 m e a colorida R6G5B4, $30 \mathrm{~m}$, permitindo alterar os valores de pixel de $30 \mathrm{~m}$ para $15 \mathrm{~m}$ e resultando numa imagem fusionada multiespectral de resolução espacial de $15 \mathrm{~m}$ com maior nitidez.

Gerou-se um buffer de $500 \mathrm{~m}$ a partir do arquivo vetorial em shapefile do limite do parque para acrescentar a região do entorno, constituído pelo arruamento e edificações e, posteriormente, as imagens foram recortadas para utilização, apenas, da área de interesse e redução do tempo de espera de processamento. 
Figura 2. Fluxograma Metodológico.

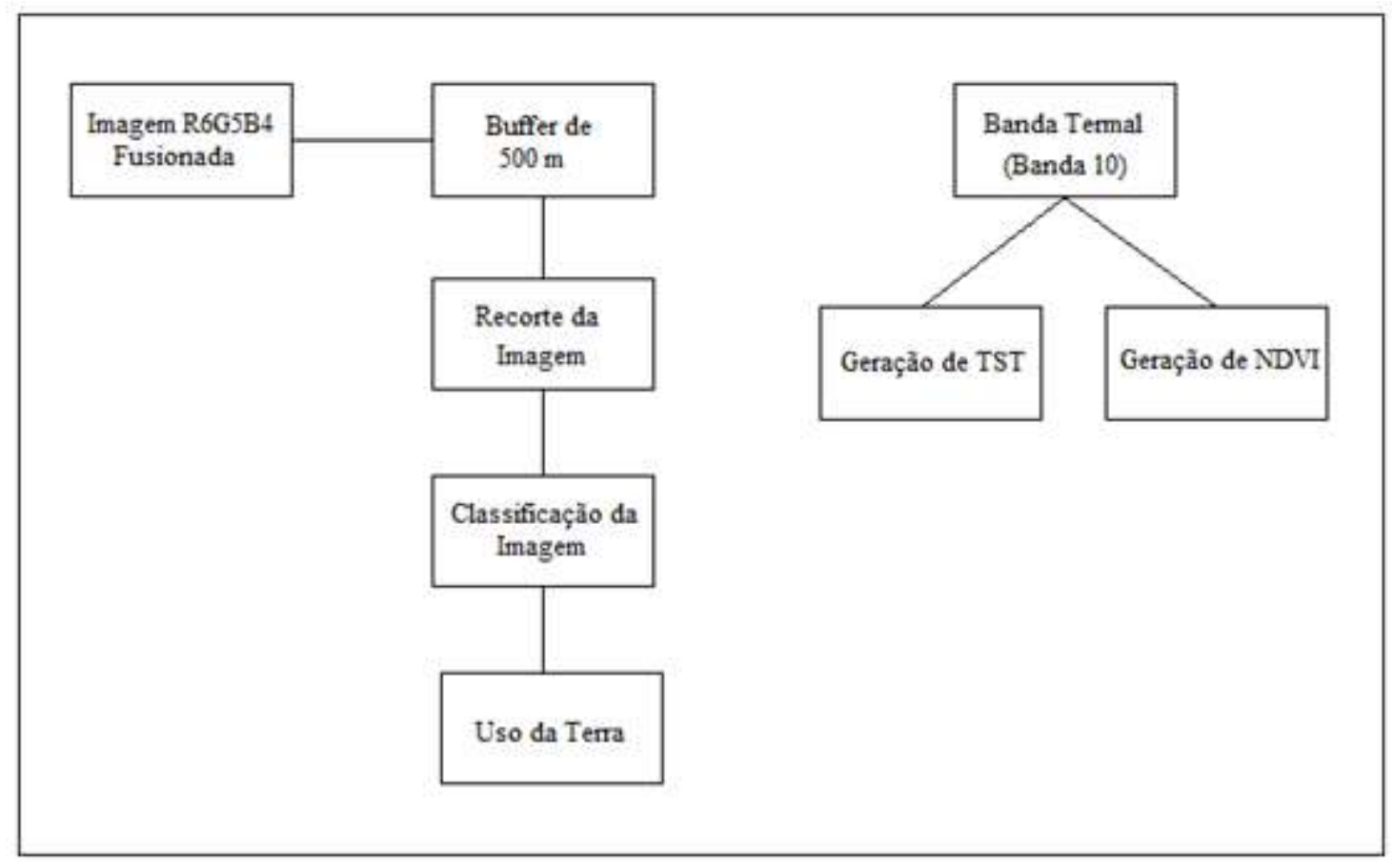

Fonte: Autores (2021).

Para o processo de classificação do uso e ocupação da terra empregou-se o método da Máxima verossimilhança (Maxver) ou maximum likehood. Nesta etapa foram adquiridas amostras de treinamento para o algoritmo citado em forma de polígonos sobre a imagem fusionada, permitindo a classificação pixel a pixel da imagem, pelo cálculo da probabilidade de um determinado pixel pertencer a uma nova classe (Leite e Rosa, 2012). O mapa gerado a partir desta classificação foi convertido de dados raster para vetoriais do tipo polígono no formato shapefile, o que possibilitou quantificar e identificar todas as classes fitofisionômicas, sendo elas: Mata de Galeria, Cerradão, Cerrado Stricto Sensu e Área Urbana.

Na obtenção da TST foi utilizado a radiância espectral $L_{\lambda}$ da banda termal com o uso da equação 1 .

$$
L_{\lambda}=M_{L} \times Q_{\text {cal }}+A_{L}
$$

equação 1

Onde: $L_{\Omega}=$ Radiância Espectral do sensor de abertura em Watts/ $(\mathrm{m} 2 \mathrm{sr} \mu \mathrm{m})$;

$M_{L}=$ Fator multiplicativo de redimensionamento da banda $10=3.3420 \mathrm{E}-04$;

$Q_{\text {cal }}=$ Valor quantizado e calibrado do pixel em nível de cinza (DN) (banda 10);

$A_{L}=$ Fator de redimensionamento aditivo específico da banda $10=0.10000$.

Segundo Pinheiro e Silva (2017), a TST em Kelvin foi calculada aplicando a equação 2 e, para transformar de Kelvin para Graus Celsius, subtraiu-se 273,15 conforme a equação 3.

$$
T_{K}=\frac{K_{2}}{\left.\ln \frac{K_{1}}{L_{2}}+1\right\}}
$$




$$
T_{C}=\frac{\mathbb{K}_{2}}{\ln \left(\frac{\mathbb{K}_{1}}{L_{\mathrm{\lambda}}}+1\right)}-273,15
$$

Onde:

$$
\begin{aligned}
& T_{K}=\text { Temperatura efetiva no satélite em Kelvin }(\mathrm{K}) ; \\
& K_{2}=\text { Constante de calibração } 2=1321,08(\mathrm{~K}) ; \\
& K_{1}=\text { Constante de calibração } 1=774,89(\mathrm{~K}) ; \\
& L_{\lambda}=\text { Radiância espectral. }
\end{aligned}
$$

O NDVI desenvolvido por Rouse et al., (1973) foi calculado a partir dos valores de reflectâncias contidas nas bandas espectrais, Banda 4 do visível e Banda 5 do infravermelho próximo, com a razão normalizada entre a diferença e a soma das referidas bandas conforme a equação 4 .

$$
\text { NDVI }=\frac{\text { Banda } 4-\text { Banda } 5}{\text { Banda } 4+\text { Banda } 5}
$$

equação 4

Onde:

$$
\begin{aligned}
& \text { NVDI = Normalized Difference Vegetation Index; } \\
& \text { Banda } 4 \text { = Visível Vermelho; } \\
& \text { Banda } 5 \text { = Infravermelho Próximo. }
\end{aligned}
$$

Este índice apresenta uma escala normalizada a razão simples entre -1 e +1 , sendo que, áreas com vegetação mais densa, consequentemente, mais vigorosa possuem valores próximos de +1 . Para regiões com cobertura vegetal menos densa, tendendo a solo exposto computam valores próximos de zero e, valores próximos de - 1 são encontrados em superfícies de corpos d'água.

\section{Resultados e Discussões}

\section{Uso e ocupação da terra}

Definiram-se as classes de uso e ocupação da terra, representadas por uma área dentro do PEMB e no seu entorno ocupado por área urbana com alta densidade de construção. Foram identificadas as classes de vegetação Mata de Galeria, Cerradão, Cerrado Stricto Sensu e Área construída, constituídas de áreas do entorno e no PEMB destinadas aos postos com equipamentos de ginástica para a prática de exercícios físicos, sanitários, bebedouros, estacionamento, mirante, centro de educação ambiental, locais para atividades sociais e administrativas e, também, as trilhas que cortam o local gerados a partir da classificação supervisionada das imagens multiespectrais, conforme demostrada na Figura 3. 
Figura 3. Distribuição das classes de uso e cobertura da terra para os anos de 2013 e 2020 nas figuras A e B, respectivamente.

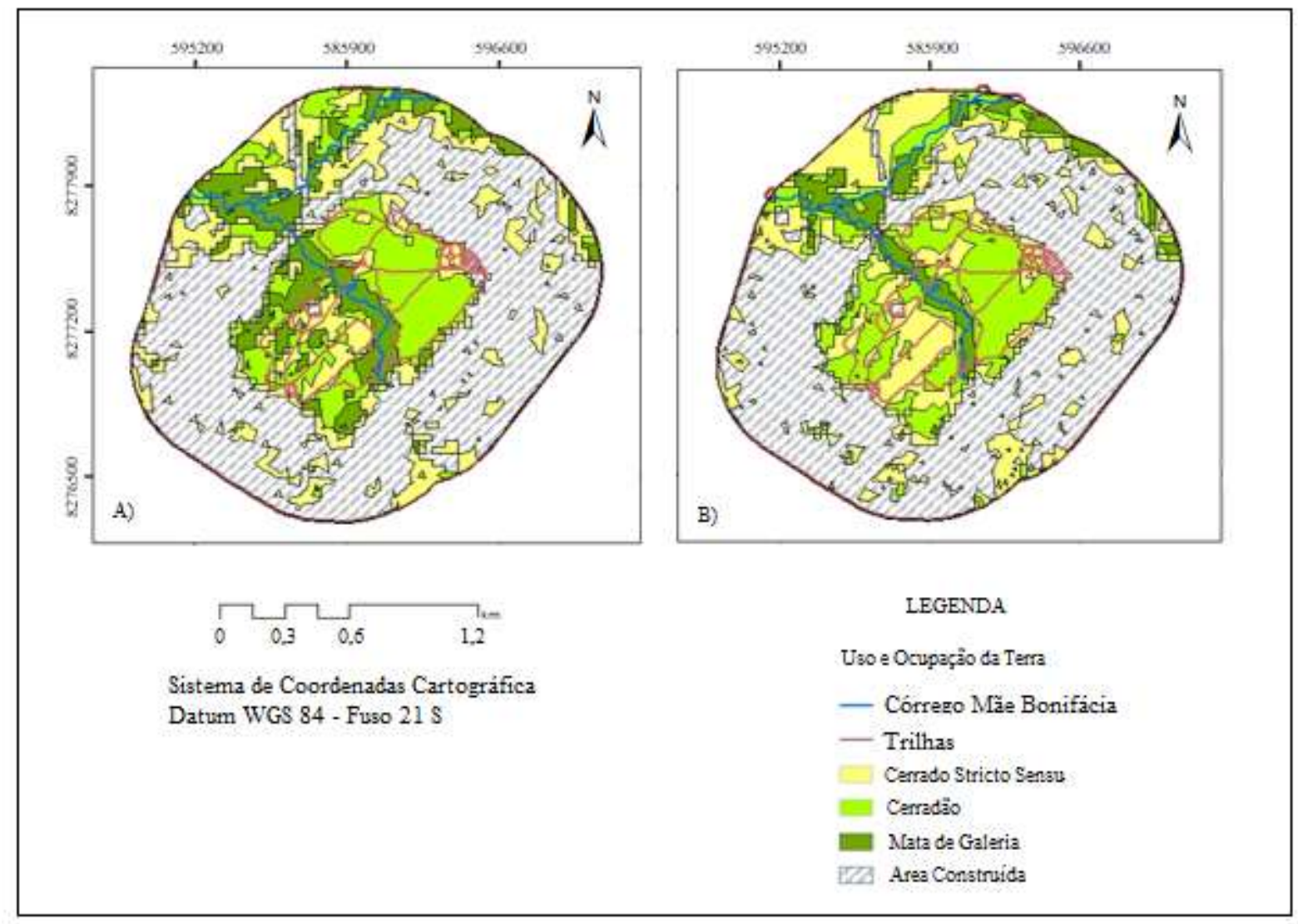

Fonte: Autores (2021).

Corroborando com esta pesquisa, Nascimento (2015) verificou que o Parque Estadual Mãe Bonifácia conta com o bioma Cerrado e uma cobertura vegetal constituída por três fisionomias distintas, sendo Mata de Galeria, que acompanha os corpos d'água geralmente com árvores de maior porte; Cerradão, que é menos denso e com árvores de médio porte; Cerrado stricto sensu: com uma vegetação um pouco mais rala e arbustiva, sendo encontrada em áreas um pouco mais elevada.

Da área total do parque de $0,775 \mathrm{Km}^{2}$, aproximadamente $0,739 \mathrm{Km}^{2}$ equivalente a $95 \%$ estão ocupadas com vegetação e $0,035 \mathrm{Km}^{2}$ ou $5 \%$ estão destinadas à classe Outros (Sema, 2013).

O Parque tem sua hidrografia restrita a um pequeno arroio que percorre sua área física, córrego Mãe Bonifácia. A área hídrica representa aproximadamente $0,42 \%$ e é responsável pela sobrevivência de diversas espécies da fauna e flora.

Os valores obtidos para cada uma das classes e seus percentuais, nos respectivos anos, são apresentados na Tabela 2. Verifica-se que a classe de maior ocupação correspondeu a área urbana com um crescimento de $1,12 \%$ de 2013 para 2020. Considerando este intervalo cronológico, ocorreram aumento das classes de Cerrado Stricto Sensu e Cerradão de 2,33\% e 2,02\%, respectivamente, enquanto, ocorreu redução da classe Mata de Galeria de 5,47\%.

Segundo Ferreira e Silva (2019) a vegetação nativa é de extrema importância para a dinâmica ambiental, tendo em vista o potencial ecológico que elas possuem, manutenção da fauna, regulador térmico, proteção para os recursos hídricos, proteção do solo, protegendo as camadas superficiais do solo de eventos erosivos, lixiviação e demais danos ambientais, sobretudo, sendo grande responsável pela manutenção das águas subterrâneas. 
Tabela 2. Classes de uso e cobertura da terra dos anos de 2013 e 2020.

\begin{tabular}{c|c|c|c|c}
\hline \multirow{2}{*}{ Classes } & \multicolumn{2}{|c|}{$\mathbf{2 0 1 3}$} & \multicolumn{2}{c}{$\mathbf{2 0 2 0}$} \\
\cline { 2 - 5 } & $\mathbf{k m}^{\mathbf{2}}$ & $\mathbf{\%}$ & $\mathbf{k m}^{\mathbf{2}}$ & $\mathbf{\%}$ \\
\hline Mata de Galeria & 0,363 & 11,15 & 0,185 & 5,68 \\
Cerradão & 0,516 & 15,85 & 0,582 & 17,87 \\
Cerrado Stricto Sensu & 0,665 & 20,43 & 0,741 & 22,76 \\
Área Construída & 1,712 & 52,58 & 1,748 & 53,68 \\
Total & 3,256 & 100,00 & 3,256 & 100,00 \\
\hline
\end{tabular}

Fonte: Autores (2021).

A Figura 4, a seguir, apresenta um quantitativo em porcentagens registradas para cada classe detectada, assim como, as variações na estrutura da paisagem ocorridas nos anos de 2013 e 2020.

Figura 4. Percentual das classes de Uso e Cobertura da Terra

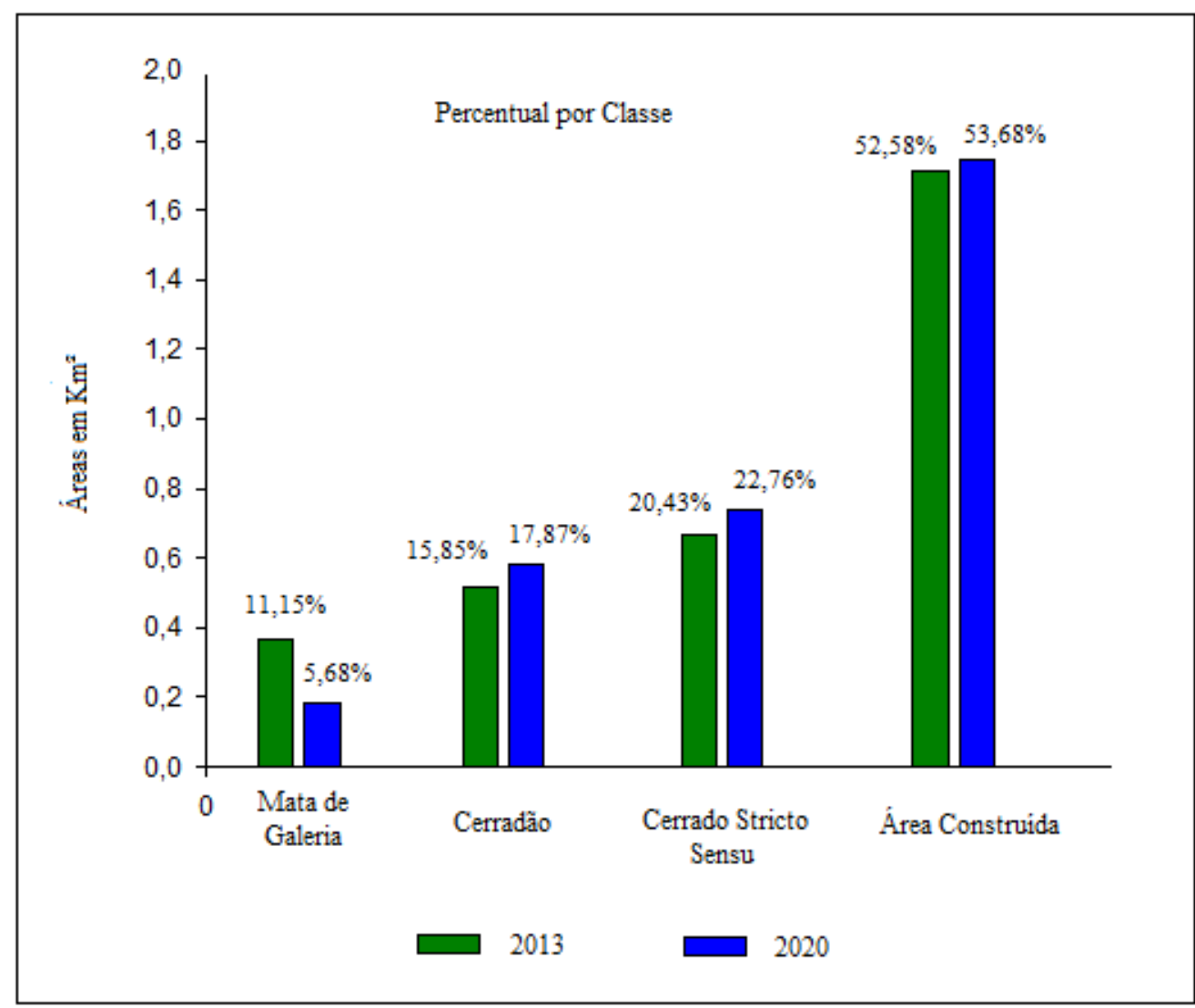

Fonte: Autores (2021).

A Figura 5, apresenta os índices de distribuição pluviométrica nos anos de 2013 e 2020. Verifica-se o valor da precipitação total anual em torno de 1.540 mm/ano em 2013, e uma queda nos meses de abril, maio, junho, julho e setembro, ocorrendo no mês de agosto um déficit hídrico, com ausência de chuvas. 
Figura 5. Distribuição da precipitação pluviométrica acumulada mensalmente nos anos de 2013 e 2020.

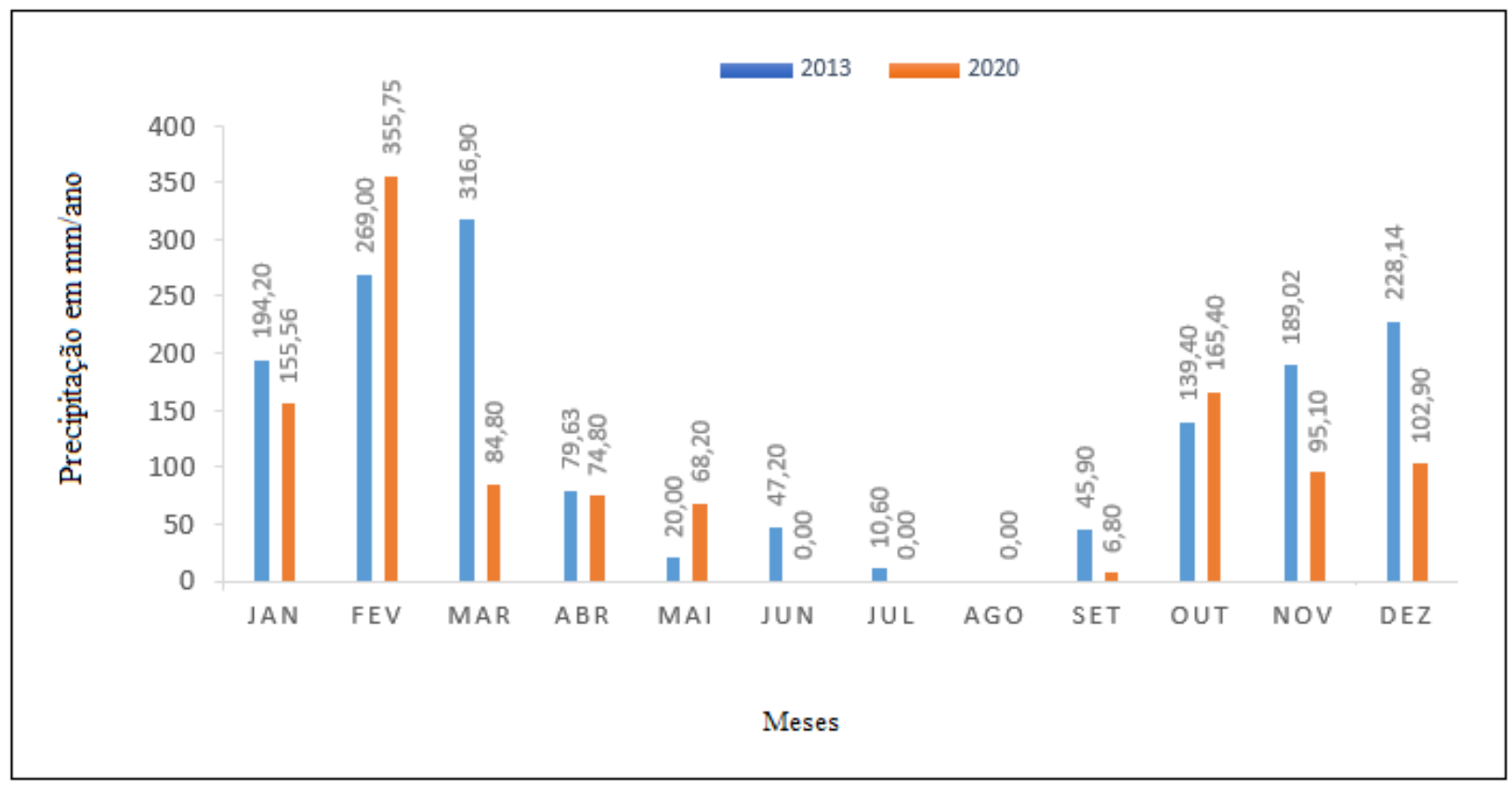

Fonte: Autores (2021).

Posteriormente, no ano de 2020 houve uma redução na distribuição da precipitação anual com 1.109,31 mm/ano. Todos os meses com exceção de fevereiro apresentaram redução hídrica, comparando-se ao ano de 2013 e os meses de junho, julho e agosto foram marcados por déficit hídrico. Confirmando esses dados, Santos (2000) cita Cuiabá tendo uma estação chuvosa que vai de outubro a abril, sendo mais intenso o período de dezembro a fevereiro e, a estação seca de maio a setembro, apresentando meses mais secos em junho, julho, agosto e setembro.

\section{Distribuição espacial da TST e do NDVI}

O cálculo da temperatura de superfície revela uma alta concentração de áreas mais quentes na região periférica do PEMB, revelando um padrão, provavelmente devido à proximidade das áreas urbanas em cada ano analisado, conforme mostra a Figura 6 e 3. As informações mostraram temperaturas proporcionais às tipologias das classes de vegetação, uma vez que menores intervalos foram registrados nas classes de Mata de Galeria, $27,99^{\circ}$ a $30,38^{\circ} \mathrm{C}$ e Cerradão, $27,99^{\circ}$ a $30,38^{\circ} \mathrm{C}$, seguida da classe Cerrado Stricto Sensu com intervalos de 27,04 a $28,23^{\circ} \mathrm{C}$ e 30,39 a $31,22^{\circ} \mathrm{C}$ em 2013 e 2020 , respectivamente. Para a classe área construída foi verificada faixas maiores de temperaturas, verificando $28,24{ }^{\circ} \mathrm{C}$ a $30,41{ }^{\circ} \mathrm{C}$ no ano de 2013 e $31,23{ }^{\circ} \mathrm{C}$ a $33,48^{\circ} \mathrm{C}$ em 2020 .

Assim, as mudanças no uso e cobertura da terra possuem uma relação direta com os valores de TST. Comparando as Figuras 6 e 3, constata-se que as temperaturas mais elevadas foram registradas nas superfícies do solo coberto por áreas pavimentadas, com a presença de vegetação esparsa como descrito para o ano de 2020 no que se refere a área urbana.

Desta forma, conforme Lombardo (1985), surgem as ilhas de calor referente as anomalias térmicas, em que, a temperatura nas áreas mais antropizadas, geralmente nos centros das grandes cidades, é maior que em áreas vegetadas. Aram et al., (2020) verificaram que o efeito de resfriamento varia com a distância do parque, observando a redução à uma distância de 520 m, quando apresenta o domínio da ilha de calor. Em estudos do microclima e conforto térmico, Cunha et al., (2017) e Melo e Dias (2019) obtiveram resultados similares, relacionando a influência da cobertura vegetal com a proximidade de áreas urbanizadas. 
Figura 6. Distribuição da TST nos anos de 2013 e 2020.

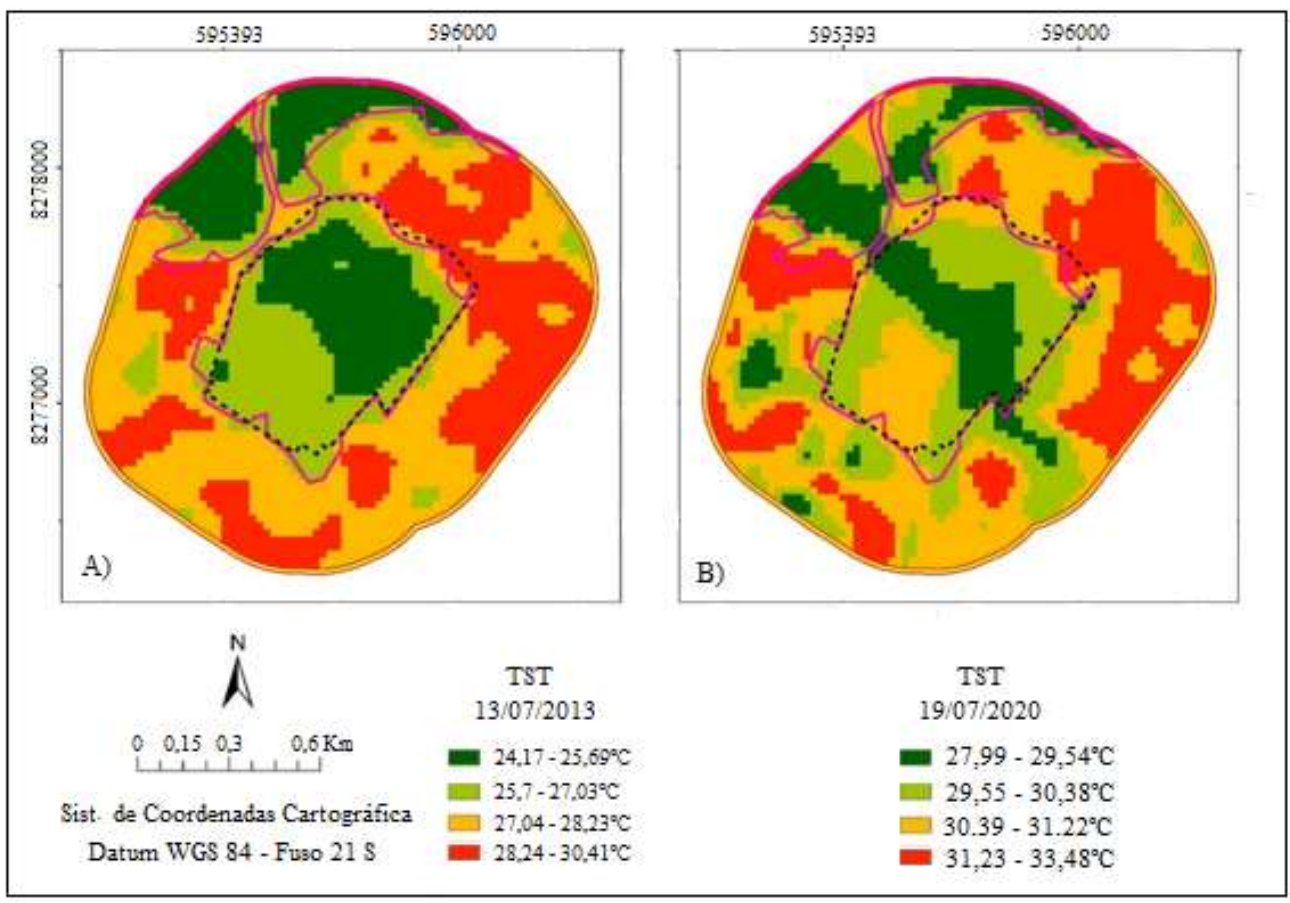

Fonte: Autores (2021).

Ao analisar o NVDI nas regiões de áreas urbanas e áreas verdes, conforme as Figuras 7 e 3, os valores mínimos de NDVI estão localizados nas porções de áreas urbanas, variando entre -0,035 e 0,22 para o ano de 2013 e -0,03 e 0,20 o ano de 2020 e valores mais elevados entre 0,23 a 0,47 e 0,21 a 0,49 para o ano 2013 e 2020, respectivamente. Estes valores, aproximam-se daqueles encontrados por Cavalcante (2020) em áreas de savanas.

Comparando os resultados obtidas de NDVI e TST, observa-se uma inversão de valores. Para altos valores de TST correspondem à baixos valores de NDVI, concentrando na porção centro-sul e a noroeste do PEMB no ano de 2013. Já em 2020 densificou-se mais a região central e a nordeste da área de estudo.

Analisando o NDVI e TST em conjunto, verifica-se que as regiões que apresentaram valores mais elevados de NDVI, sobrepuseram, aproximadamente, às áreas com distribuição de baixa TST, configurando em um ambiente com melhor conforto ambiental e que contribui para a redução das temperaturas. Situações contrárias podem servir como informações úteis aos gestores ambientais, na tomada de decisão mais assertiva, a exemplo do uso adequado da terra. 
Figura 7. Distribuição do NDVI nos anos de 2013 e 2020.

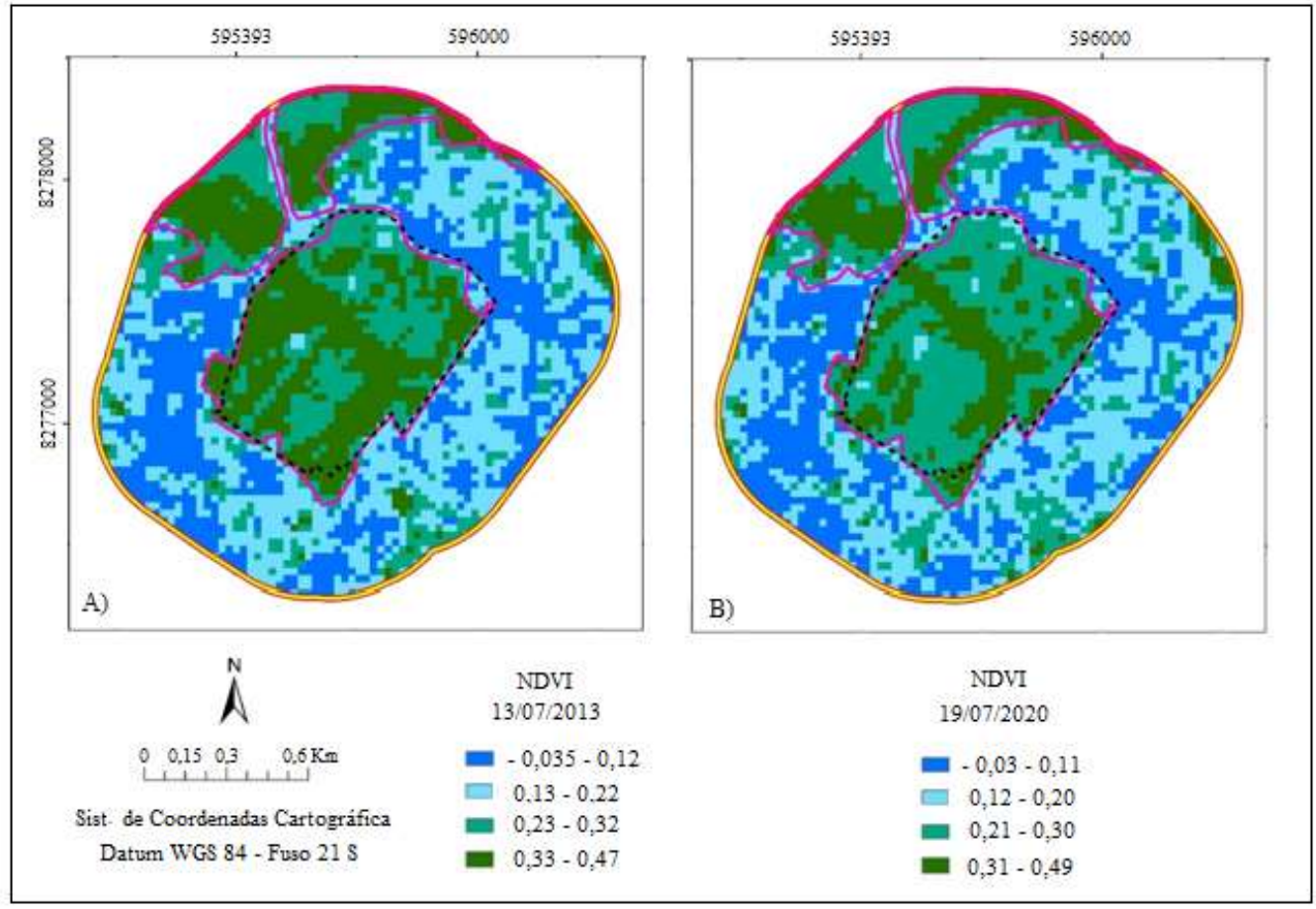

Fonte: Autores (2021).

\section{Conclusão}

As imagens de satélite Landsat 8 e SIG possibilitam um conhecimento da superfície terrestre onde foi possível estudar e extrair as informações biofísicas da TST e do NDVI e a relação entre eles no estudo do uso e cobertura da terra e suas relações com o microclima no centro urbano da cidade de Cuiabá. Os resultados mostram, a partir da metodologia adotada, relações inversas entre os valores de NDVI e TST no período considerado, destacando regiões coberta por vegetação mais densa, valores mais elevados do NDVI e temperaturas reduzidas.

Estudos desta natureza vem sendo adotados nas áreas urbanas como forma de minimizar os impactos ambientais e incentivar a implantação ou preservação do espaço com vegetação, visto que que as plantas reduzem o aquecimento e potencializam a qualidade de vida da população.

Assim, no presente trabalho, verifica-se que o efeito de resfriamento do Parque Mãe Bonifácia é maior nas áreas com valores mais elevados de NVDI, evidenciando-se a importância das áreas de matas no interior do PEMB e no seu entorno, ressaltando que as temperaturas mais elevadas, concentram-se no perímetro urbano, com variações consideráveis nos anos estudados e intensificadas pelo déficit hídrico acorrido no ano de 2020.

Como sugestões de trabalho futuros que promovam resultados mais concisos e mais assertivos seria a adoção de uma quantidade maior de anos a serem analisados e a utilização de imagens de melhor resolução espacial.

\section{Referências}

Aram, F., Solgi, E., Garcia, E. H., \& Mosavi, A. (2020). Urban heat resilience at the time of global warming: evaluating the impact of the urban parks on outdoor thermal comfort. Environmental sciences Europe, 32 (1), 1-15.

Azambuja, W. A, Bombassaro, M, Zolin, T. D. S, \& Ribeiro, V. O. (2018). Analise da relação da temperatura de superficie e indice de vegetação (NDVI) para Dourados-MS. ABES - Associação Brasileira de Engenharia Sanitária e Ambiental. IN: XIV SIBESA. Foz do Iguaçu, Paraná, Brasil.

Barros, A. S, Farias, L. M, \& Marinho, J. L. A. (2020). Aplicação do Índice de Vegetação por Diferença Normalizada (NDVI) na Caracterização da Cobertura Vegetativa de Juazeiro Do Norte - CE. Revista Brasileira de Geografia Física, 13(6), 2885-2895. 
Cuiabá. (2004). Legislação Urbana de Cuiabá. Instituto de Pesquisa e Desenvolvimento Urbano. https://www.cuiaba.mt.gov.br.

Cavalcante, J. C. Almeida, C. A, \& Castelo, T. B. (2020). Análise de NDVI e temperatura da superfície terrestre utilizando a plataforma do google earth engine em área de savana amazônica e floresta secundária, no município de Vigia - PA. Northeast Geosciences Journal. 6(1), 65 - 70.

Cuiabá. (2021). Pontos Turísticos: Parque Mãe Bonifácia. Camara Municipalde Cuiabá. http://www.camaracuiaba.mt.gov.br/.

Cunha, M. I. A, Marcilio, N. S. A, Rausch, P. M, \& Koniski, M. C. (2017). Imagem termal do Landsat 8/TIRS/OLI: análise da temperatura de parques urbanos. In: Anais do XVIII Simpósio Brasileiro de Sensoriamento Remoto-SBSR. Santos, São Paulo, Brasil.

Duarte, D. H. S. (2010). Variáveis Urbanísticas e Microclimas Urbanos - Modelo Empírico e Proposta de um Indicador. Forum Patrimônio: amb. constr. e patr. Sustentável, 3(2), 3 - 37.

Eduvirgem, R. V, Periçato, A. J, Santos, M. V, \& Caraminan, L. M. (2019). Investigação da temperatura aparente de superfície e do índice de vegetação por diferença normalizada da zona urbana de Cascavel, PR. Geofronter, 5(4), 51-71. https://periodicosonline.uems.br/index.php/GEOF/index.

IPDU. 2012. Perfil Socioeconomico de Cuiabá. Instituto de Planejamento e Desenvolvimento Urbano. $2012 . \quad$ (IV), 58. https://www.cuiaba.mt.gov.br/upload/arquivo/perfil socioeconomico_de_cuiaba_Vol_IV.pdf.

Leite, E. F., \& Rosa, R. (2012). Análise do uso, ocupação e cobertura da terra da bacia hidrográfica do Rio Formiga, Tocantins. Revista Eletrônica de Geografia, 4(12), 90-106.

Lombardo, M. A. (1985). Ilha de Calor nas Metrópoles: o Exemplo de São Paulo. 244p.

Lopes, E. R, N., Silva, A. P, P., Peruchi, J. F., \& Lourenço, R. W. (2018). Zoneamento de Risco de Incêndio e Queimadas no Município de Sorocaba. Revista do departamento de geografia, 36, p. 118-129.

Martins, M. C. (2018). Revisão teórica e discussões sobre o uso e ocupação do solo no clima urbano de juiz de fora. Monografia do curso de especialização em Sistemas Tecnológicos e Sustentabilidade Aplicados ao Ambiente Construído. Universidade federal de Minas Gerais (UFMG).

Melo, B. M, \& Dias, D. P. (2019). Microclima e conforto térmico de remanescentes florestais urbanos no município de Jataí - GO. Revista REVSBAU, 14(2), $01-15$.

Martins, E. C, \& Romancini, S. R. (2005). Natureza na cidade: um olhar sobre o parque Mãe Bonifácia - Cuiabá/MT. http://observatoriogeograficoamericalatin a.org.mx/egal10/Procesosambientales/Proteccioncivil/01.pdf.

Monteiro, C. A. F.2015. Clima Urbano.

Mwangi, P.W., Karanja, F. N., \& Kamau, P.K. Analysis of the Relationship between Land Surface Temperature and Vegetation and Built-Up Indices in Upper-Hill, Nairobi. Journal of Geoscience and Environment Protection, (6), 1-16.

Ochtyra, A., Marcinkowska-Ochtyra, A., \& Raczko, E., (2020). Threshold-and trend-based vegetation change monitoring algorithm based on the inter-annual multi-temporal normalized difference moisture index series: A case study of the Tatra Mountains. Remote Sensing of Environment [online] 249. https://doi.org/10.1016/j.rse.2020.112026.

Oliveira, P., \& Bitar, O. (2009). Indicadores Ambientais para o monitoramento de parques urbanos. Revista de Gestão Integrada em Saúde do Trabalho e Meio Ambiente, 4(2), 2 - 14.

ONU (Organização das nações Unidas). 2021. http:// www.unric.org/pt/actualidade/31537-relatorio-da-onu-mostra-população-mundialcada-> .

Pinheiro, M. A, \& Silva, J. M. O. (2019). Análise da temperatura de superfície e a relação com o NDVI da zona urbana do município do Crato/Ceará 2017. Revista da Casa da Geografia de Sobral, 21(2), 334-347.

Rodrigues, A. P. M, Pasqualetto, A, \& Garção, A. L. O. (2017). A Influência dos arques urbanos no microclima de Goiânia. Revista Baru, 3(1), 25-44.

Rouse, J.W, Haas, R.H, Schell, J. A, \& Deering, D. W. (1973).Third ERTS Symposium, 1973. Monitoring vegetation systems in the Great Plains with ERTS. $N A S A$, v. I, 309-317.

SEMA - (Secretaria Estadual do Meio Ambiente). 2013. Plano de Manejo do Parque Estadual Mãe Bonifácia. https://documentacao.socioambiental. org/ato_normativo/UC/2089_20160309_181503.df.

Santos, M. V., Relatório Técnico Consolidado de clima para o Estado de Mato Grosso, 2 (2): Sistematização das Informações Temáticas nível compilatório, 2000. http://www.dados.mt.gov.br/publicacoes/dsee/climatologia/rt/DSEE-CL -RT-002.

Silva, J. S., \& Silva, R. M. D. (2016). Estimativa da temperatura de superfície e ilhas de calor urbanas na bacia do rio Bacanga, São Luís (MA). In: Simpósio Brasileiro de Climatologia Geográfica, Variabilidade e susceptibilidade climática: Implicações Ecossistêmicas e Sociais (SBCG), Goiânia, Goiás, Brasil.

Vermote, E., Justice, C., \& Claverie, M., Franch, B. (2016). Preliminary analysis of the performance of the Landsat 8/OLI land surface reflectance product. Remote Sensing of Environment, (185), 46-56.

Yang, C, He, X., Yu, L., Yang, J., Yan, F., Bu, K., Chang, L., \& Zhang, S. (2017). The Cooling Effect of Urban Parks and Its Monthly Variations in a Snow Climate City. Remote Sensing. 9(1066), 2 - 17.

Wall, E, \& Waterman, T. Desenho Urbano.2012. Bookman. 